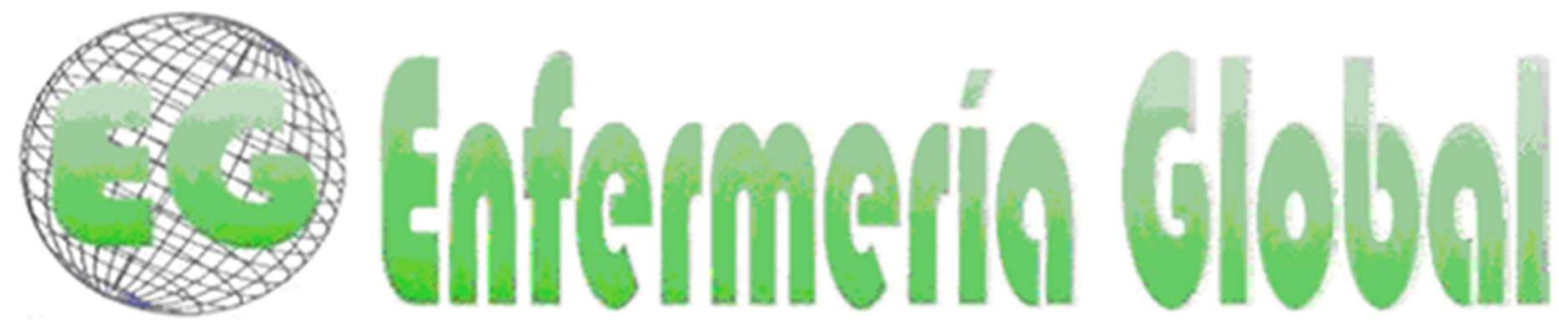

www.um.es/egloball

Enero 2021

\title{
ORIGINALES
}

\section{Validación de un instrumento para evaluar el componente comunitario de la estrategia de atención integrada a las enfermedades prevalentes de la infancia (AIEPI)}

Validation of an instrument to evaluate the community component of the integrated care strategy for prevalent childhood diseases (IMCI)

Jacqueline Hernández-Escolar ${ }^{1}$

Cristina Bohórquez Moreno

Sandra Mondragón- Bohórquez ${ }^{3}$

\begin{abstract}
${ }^{1}$ Bacterióloga, Magíster en Salud Pública, Grupo de Investigación Salud y Prácticas Sociales-SYPRES, Universidad de San Buenaventura, Cartagena, Colombia. jhernandez@usbctg.edu.co

2 Enfermera, Magister en Farmacología. Docente programa de Enfermería, Corporación Universitaria Rafael Núñez- Barranquilla. Colombia.

${ }^{3}$ Fonoaudióloga, Magister en trastornos del lenguaje y del habla. Grupo de Investigación Salud y Prácticas Sociales-SYPRES, Universidad de San Buenaventura, Cartagena, Colombia.
\end{abstract}

\section{https://doi.org/10.6018/eglobal.422001}

Recibido: 6/04/2020

Aceptado: 5/07/2020

\section{RESUMEN:}

Objetivo: Validar un instrumento para evaluar el componente comunitario, de la estrategia de atención integrada a las enfermedades prevalentes de la infancia (AIEPI).

Método: Estudio transversal descriptivo, el cual buscó hallar la validez de constructo de la encuesta SIAC utilizada para medir AIEPI comunitario, mediante un análisis factorial exploratorio, el cual redujo el número de ítems que explicaban los constructos; participaron 120 madres adolescentes de la ciudad de Cartagena, Colombia. La validación de constructo se realizó a través del Análisis Factorial Exploratorio, con el método de Factores Principales, se utilizó la prueba de esfericidad de Bartlett y la medida de la adecuación muestral de Kaiser-Meyer-Olkin (Coeficiente KMO) mediante el paquete estadístico SPSS versión 22.0.

Resultados. De acuerdo a los valores obtenidos en los dominios 1 (saneamiento básico), 3 (lactancia materna y alimentación complementaria), 4 (inmunizaciones), 5 (signos de alarma), 11 (cuidado de la mujer gestante) y 13 (SIDA), se procedió a aplicar el método de factorización por ejes principales. En el dominio 7 (prevención de accidentes) se realizó el método de factorización por componentes principales.

Conclusiones: La encuesta SIAC mantuvo en los 7 dominios buenos niveles de validez después del cambio, se hace necesario evaluar los puntos de corte que permitan un diagnóstico más sensible y específico del grado de apropiación y aplicación de las practicas clave de la estrategia AIEPI, por parte de los cuidadores de los niños y niñas menores de 5 años.

Palabras clave: Atención Integrada a las Enfermedades Prevalentes de la Infancia, niño, madres, Validez. 


\begin{abstract}
:
Objective: Validate an instrument to evaluate the community component of the integrated care strategy for prevalent childhood diseases (IMCl).

Method: descriptive cross-sectional study, which sought to find the construct validity of the SIAC survey specified to measure community $\mathrm{IMCl}$, through an exploratory factor analysis, which reduces the number of items that explained the constructs; 120 teenage mothers from the city of Cartagena, Colombia participated. The construction validation was carried out through the Exploratory Factor Analysis, with the Main Factors method, the Bartlett sphericity test and the measurement of the sampling adequacy of Kaiser-Meyer-Olkin (KMO coefficient) were performed using the SPSS statistical package. version 22.0.

Results: According to the values obtained in domains 1 (basic sanitation), 3 (breastfeeding and complementary feeding), 4 (immunizations), 5 (warning signs), 11 (care of pregnant women) and 13 (AIDS), the main axis factorization method was applied. In domain 7 (accident prevention) the principal component factorization method was performed.

Conclusions: the SIAC survey maintained good levels of validity after the change in the 7 domains, it is necessary to evaluate the cut-off points that allow a more sensitive and specific diagnosis of the degree of appropriation and application of the key practices of the IMCl strategy, for part of the caregivers of children under 5 years
\end{abstract}

Key words: Integrated Management of Childhood IIIness, child, mother, Validity.

\title{
INTRODUCCIÓN
}

La estrategia AIEPI es definida como la Atención Integrada a las Enfermedades Prevalentes de la Infancia, es promovida en el ámbito internacional por la Organización Panamericana De la Salud (OPS), la Organización Mundial de la Salud (OMS) y el Fondo de las Naciones Unidas para la Infancia (UNICEF) (1). Contiene medidas de promoción, prevención, diagnóstico y tratamiento de las enfermedades que se presentan con mayor prevalencia en la infancia, tales como: Infección Respiratoria Aguda (IRA), Enfermedad Diarreica Aguda (EDA), desnutrición, dengue, malaria, enfermedades inmunoprevenibles, maltrato infantil y alteraciones del crecimiento y desarrollo ${ }^{(2)}$.

El objetivo de la estrategia es ayudar a reducir la morbilidad y mortalidad infantil debido a que estas enfermedades son fácilmente prevenibles, favoreciendo de este modo el crecimiento y desarrollo saludable en niños menores de cinco años, especialmente de los grupos más vulnerables, buscando a través de ella, el desarrollo participativo de proyectos en áreas de alto riesgo, para apoyar la efectiva aplicación de las prácticas claves ${ }^{(3)}$.

La estrategia ha sido implementada en diferentes países del mundo, en China, por ejemplo, ha tenido un gran impacto en el sistema de salud puesto que luego de su ejecución, se aplicaron encuestas adaptadas a dicha población, donde se pudo evidenciar mejorías en los hospitales y sitios de atención a los niños ${ }^{(4) .}$

En las experiencias de la implementación de la estrategia AIEPI en América Latina, se ha utilizado una encuesta de base, que ha permitido evidenciar el conocimiento de padres y cuidadores sobre las prácticas de la misma. Esta encuesta llamada Sistema de Información de AIEPI Comunitario (SIAC) fue realizada por la OPS, la OMS y la UNICEF para medir la estrategia, a fin de conocer su efectividad e identificar la persistencia de prácticas inadecuadas de mujeres gestantes y de cuidadores que puedan afectar la salud de niños y niñas menores de 5 años ${ }^{(5)}$. 
En Colombia el componente comunitario de la estrategia AIEPI se ha llevado a cabo desde el año 2007, aplicándose encuestas no validadas para identificar los problemas entorno a la salud de los menores de cinco años y de las madres gestantes e intervenirlos ${ }^{(6)}$.

La encuesta SIAC fue creada para evaluar la efectividad de la estrategia AIEPI, no se ha encontrado evidencia sobre estudios que aborden la validez de constructo de la misma, y a través de esta operación se podrá determinar si es un instrumento confiable y fiable para medir el grado de apropiación que tienen las madres sobre las prácticas trabajadas a través del componente de AIEPI comunitario. La validación de un instrumento es importante para determinar la calidad de medición del mismo, una de las características esenciales de esta, es permitir evidenciar que el instrumento mide el constructo para el cual fue diseñado ${ }^{(7)}$.

Por tanto, el propósito de este estudio fue validar la encuesta SIAC en una población de madres adolescentes, utilizando para ello un análisis factorial exploratorio.

\section{MATERIALES Y METODOS}

\section{Diseño y muestra}

Se realizó un estudio transversal descriptivo, el cual buscó hallar la validez de constructo de la encuesta SIAC, mediante un análisis factorial exploratorio que logró reducir el número de ítems que explicaban los constructos en la encuesta original.

La población estuvo conformada por 120 madres adolescentes institucionalizadas en la Fundación Juan Felipe Gómez Escobar, en la ciudad de Cartagena, Colombia, las cuales accedieron de forma voluntaria a participar en el estudio, previa firma de consentimiento informado. En este lugar, es aplicada la estrategia AIEPI; en cuanto a la edad de las madres, esta osciló entre los 16 y 24 años, para las menores de edad, se obtuvo el consentimiento informado por parte de sus padres o acudiente.

Esta investigación fue avalada por el comité de bioética de la Universidad de San Buenaventura de Cartagena.

\section{Instrumento}

En esta investigación se utilizó la encuesta SIAC la cual es utilizada para medir AIEPI comunitario, esta fue creada por la OPS, OMS y la UNICEF, el instrumento se encuentra abierto a adaptaciones y validaciones. Evalúa 14 prácticas claves, a través de 174 preguntas y busca medir los conocimientos, actitudes y prácticas que aplican los cuidadores. Dado que la población se encontraba institucionalizada, se seleccionaron las preguntas que no requerían observación y verificación en el hogar, a fin de tener un instrumento que pudiese hacer esta medición en poblaciones institucionalizadas.

También fueron excluidas prácticas que no eran abordadas en la fundación, por lo que resultó para validación un instrumento con un total de 9 prácticas evaluadas por 106 preguntas, manteniendo las opciones de, respuestas múltiples, preguntas con única respuesta, y saltos de preguntas. 
TABLA 1. Numero de ítems aplicados en la prueba piloto.

\begin{tabular}{|c|l|c|}
\hline Dominio & \multicolumn{1}{|c|}{ Dimensión } & Piloto \\
\hline 1 & Medidas de higiene y saneamiento & 12 \\
\hline 2 & Cuidado del niño & 3 \\
\hline 3 & Lactancia materna y alimentación complementaria & 26 \\
\hline 4 & Inmunizaciones & 12 \\
\hline 5 & Signos de alarma & 21 \\
\hline 6 & Alimentación del niño enfermo & 4 \\
\hline 7 & Prevención de accidentes & 5 \\
\hline 11 & Cuidado de la mujer gestante & 18 \\
\hline 13 & VIH /SIDA & 5 \\
\hline
\end{tabular}

\section{MÉTODO}

Se realizó una validación de constructo a través de un Análisis Factorial Exploratorio, con el método de Factores Principales, mediante el paquete estadístico SPSS versión 22.0, se estableció la matriz de correlación policórica de los ítems que constituían las preguntas del cuestionario.

Posterior al análisis de factores principales, se continuó el análisis, a través del método de factorización de ejes principales; para comprobar la adecuación de la muestra al análisis factorial, se utilizó la prueba de esfericidad de Bartlett, la cual contrasta si la matriz de correlaciones es una matriz de identidad, así mismo para contrastar si las correlaciones parciales entre las variables eran pequeñas, se tomó la medida de la adecuación muestral de Kaiser-Meyer-Olkin (Coeficiente KMO).

Al revisar la composición de los dominios hallados, posterior a la extracción se redujeron a factores que median de manera individual un comportamiento saludable. Sin embargo, se encontró que el dominio 2 (cuidado del niño) y dominio 6 (alimentación del niño enfermo), en algunos ítems no confluyeron o no fueron suficientes para medir los componentes, que según la encuesta original deberían ser observados, lo cual no los hizo aptos para su validación y fueron excluidos del análisis.

El análisis se desarrolló de forma individual para cada domino, evaluando un instrumento conformado por 9 dominios y 106 variables, las cuales después de la extracción se redujeron a 7 dominios y 24 variables, a partir de la escala original.

\section{RESULTADOS}

El análisis se desarrolló de forma individual para cada dominio, dado que la encuesta se estructura por prácticas claves, las cuales son diferentes conceptualmente entre sí, por ello, se hizo necesario validar por separado cada constructo.

En primer lugar, para comprobar la adecuación de la muestra al análisis factorial, se utilizó la prueba de esfericidad de Bartlett y la medida de la adecuación muestral de Kaiser-Meyer-Olkin (Coeficiente KMO). De acuerdo con los valores obtenidos en los dominios 1 (saneamiento básico), 3 (lactancia materna y alimentación complementaria), 4 (inmunizaciones), 5 (signos de alarma), 11 (cuidado 
de la mujer gestante) y 13 (VIH/SIDA), se procedió a aplicar el método de factorización por ejes principales. En el dominio 7 (prevención de accidentes) se realizó el método de factorización por componentes principales (Ver tabla 2).

Se evidenció que la estructura factorial de la encuesta SIAC, adaptada al castellano, en una muestra de madres adolescentes adscritas a la Fundación Juan Felipe Gómez Escobar de la ciudad de Cartagena, Colombia, fue inicialmente estructurada con 9 dominios (saneamiento básico, cuidado del niño, lactancia materna y alimentación complementaria, inmunizaciones, signos de alarma, alimentación del niño enfermo, prevención de accidentes, cuidados de la mujer gestante y SIDA) y 106 variables iniciales, información tomada de la encuesta original. Sin embargo, posterior a la extracción, se encontró que el dominio 2 (cuidado del niño) y dominio 6 (alimentación del niño enfermo), en algunos ítems no confluyeron o no fueron suficientes para medir los componentes, que según la encuesta original deberían ser observados, lo cual no los hizo aptos para su validación y fueron excluidos del análisis, quedando entonces un instrumento con 7 dominios.

TABLA 2. Valores de la prueba es esfericidad y adecuación muestral

\begin{tabular}{|c|c|c|c|}
\hline Dominio & Coeficiente KMO & \multicolumn{2}{|c|}{$\begin{array}{c}\text { Prueba de Esfericidad } \\
\text { de Bartlett }\end{array}$} \\
\hline $\begin{array}{c}1 \\
\text { Medidas de Higiene y } \\
\text { Saneamiento Básico }\end{array}$ & 0,411 & \begin{tabular}{|c|} 
Chi- \\
cuadrado \\
aproximado \\
gl \\
Sig.
\end{tabular} & $\begin{array}{c}110,486 \\
55 \\
0\end{array}$ \\
\hline $\begin{array}{c}3 \\
\text { Lactancia Materna y } \\
\text { Alimentación complementaria }\end{array}$ & ,429 & $\begin{array}{c}\text { Chi- } \\
\text { cuadrado } \\
\text { aproximado } \\
\text { gl } \\
\text { Sig. } \\
\end{array}$ & $\begin{array}{c}246,370 \\
171 \\
, 000\end{array}$ \\
\hline $\begin{array}{c}4 \\
\text { Inmunizaciones }\end{array}$ & ,505 & $\begin{array}{c}\text { Chi- } \\
\text { cuadrado } \\
\text { aproximado } \\
\text { gl } \\
\text { Sig. } \\
\end{array}$ & $\begin{array}{c}55,040 \\
28 \\
, 002\end{array}$ \\
\hline $\begin{array}{c}5 \\
\text { Signos de Alarma }\end{array}$ & 2,572 & $\begin{array}{c}\text { Chi- } \\
\text { cuadrado } \\
\text { aproximado } \\
\text { gl } \\
\text { Sig. } \\
\end{array}$ & $\begin{array}{c}402,211 \\
210 \\
, 000\end{array}$ \\
\hline $\begin{array}{c}7 \\
\text { Prevención de accidentes }\end{array}$ & 2,644 & $\begin{array}{c}\text { Chi- } \\
\text { cuadrado } \\
\text { aproximado } \\
\text { gl } \\
\text { Sig. } \\
\end{array}$ & $\begin{array}{c}50,030 \\
10 \\
, 000\end{array}$ \\
\hline $\begin{array}{c}11 \\
\text { Cuidado de la mujer gestante }\end{array}$ & ב,471 & $\begin{array}{c}\text { Chi- } \\
\text { cuadrado } \\
\text { aproximado } \\
\text { gl } \\
\text { Sig. }\end{array}$ & $\begin{array}{c}184,662 \\
105 \\
, 000\end{array}$ \\
\hline
\end{tabular}




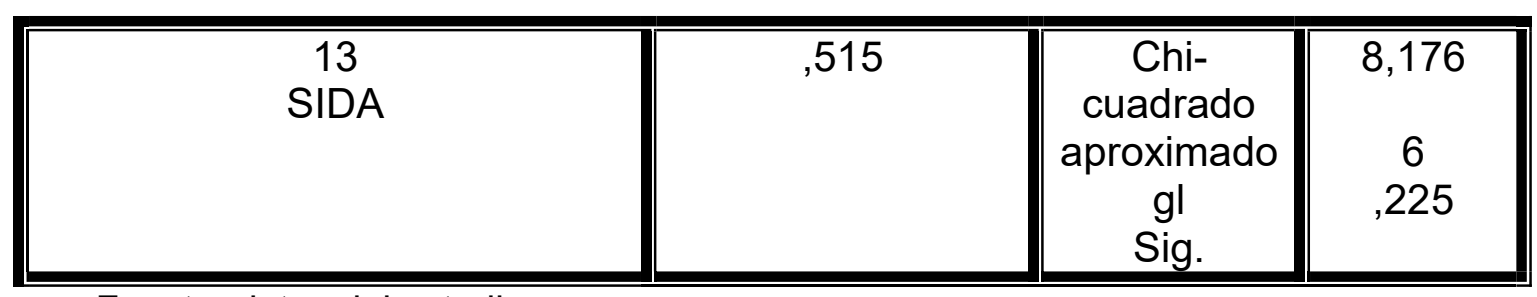

Fuente: datos del estudio

Para el dominio 1 el cual evalúa saneamiento básico, los resultados del análisis factorial mostraron una solución de 6 factores para explicar el 70,7\% de la varianza de los datos y el ajuste al modelo teórico; sin embargo al realizar la matriz de factores rotados, se observó que 5 factores aportaban el mayor peso dentro de la matriz, los cuales correspondían a las preguntas que indagan sobre: problemas por no beber agua segura, manejo de las heces del bebé, lavado de manos, manejo de basuras, consumo de agua segura en la comunidad y manejo de agua para el consumo del bebe.

Para el dominio 3 relacionado con lactancia materna y alimentación complementaria, el análisis factorial mostró que una solución de 8 factores fue la mejor en explicar el $71,9 \%$ de la varianza de los datos, reduciendo la dimensionalidad del problema de 19 a 8 factores, sin embargo al realizar la extracción por ejes principales se obtuvo un total de 4 factores (relacionados con el tiempo de lactancia después de nacer él bebé, con lo primero en dar al bebé después de nacer, el conocimiento de la vitamina $\mathrm{A}$ y en qué alimentos se podría encontrar).

En el dominio 4 relacionado con inmunizaciones, el análisis factorial arrojó que 5 factores explican el $82 \%$ de la varianza de los datos, sin embargo, al realizar el gráfico de las variables en el espacio rotado se obtuvo un total de 3 factores seleccionados, relacionados con la edad de aplicación de las primeras vacunas, cumplimiento del esquema de vacunación completo y esquema de vacunación durante el embarazo.

En el dominio 5 correspondiente a la práctica clave de signos de alarma, el análisis factorial arrojo que 7 factores explicaban el $70,8 \%$ de la varianza, posteriormente al realizar la rotación de factores este número se redujo a 5 , es el caso de las variables relacionadas con las señales que indican cuando buscar ayuda en caso de que el menor presente diarrea, que hacer en caso de presentar diarrea, a quien acudir en caso de evidenciar signos de alarma frente a la diarrea.

El análisis factorial del dominio 7 permitió evidenciar que la práctica de prevención de accidentes era explicada por 3 factores con el $83 \%$ de la varianza, al realizar la matriz de factores rotados finalmente dos explicaron mejor el dominio, estos hacen referencia a los accidentes más comunes en la comunidad y la prevención de estos.

El dominio 11 cuidado de la mujer gestante, se redujo a 7 ítems que explicaban el $75 \%$ de la varianza, posteriormente a través del gráfico de sedimentación se encontró que 4 factores explicaban el dominio, los cuales hacen referencia a la edad en que la mujer debe tener su primer hijo, quién realizó el control durante el embarazo, quién atendió el parto y la presencia de complicaciones durante el parto.

Por último, el análisis factorial del dominio 13, evidenció 3 factores que explicaban el $84 \%$ total de la varianza, posteriormente se rotaron los factores quedando las 
variables referidas al medio de trasmisión del VIH/SIDA y la relacionada con la prevención del VIH/SIDA.

Posterior al análisis que se desarrolló de manera individual para cada domino y después de la extracción, la encuesta se redujo a un instrumento que con 7 dominios y 24 variables, permite medir los constructos de la prácticas clave de la estrategia AIEPI Comunitario.

\section{DISCUSIÓN}

Mediante la siguiente investigación se realizó la validación de la encuesta SIAC, utilizada para evaluar la aplicación del AIEPI comunitario, lo que se convierte en un instrumento importante para los profesionales de salud, teniendo en cuenta que la Organización Mundial de la Salud reporta evidencias que resaltan el papel que cumple el trabajo con los padres y cuidadores de los menores de 5 años en torno al cambio de sus conocimientos, actitudes y prácticas de crianza, las cuales influyen de manera directa en el estado general de salud y de bienestar en la primera infancia. Dado que el desarrollo de prácticas inadecuadas puede impactar en el aumento de la morbilidad infantil, retrasos en el desarrollo, en el crecimiento físico, a nivel motor, cognitivo y lingüístico ${ }^{(8)}$.

Esta investigación se realizó con el fin de determinar la validez de constructo de un instrumento que evalúa 7 practicas clave de la estrategia AIEPI en su componente comunitario, y de esta forma poder medir los conocimientos, actitudes y prácticas en la población intervenida, esto se puede realizar utilizando instrumentos que cumplan con el objetivo de lo que se pretende evaluar, además de estar validados, para poder obtener resultados cercanos a la realidad de las prácticas evaluadas ${ }^{(9)}$.

En este sentido, las habilidades de las personas a cargo del cuidado en el hogar de los menores forman parte de los indicadores básicos para asegurar la continuidad y seguridad del cuidado, previniendo la morbilidad y mortalidad infantil. Por tanto, al contar con un instrumento que involucra diversas dimensiones para identificar de manera específica la competencia para el cuidado en el hogar en cuidadores y familiares de niños menores de 5 años, y que a su vez permite la identificación de necesidades propias de los cuidadores en el contexto local, con adecuados procesos de validez, se convierte en una herramienta vital para consolidar estrategias de intervención efectivas ${ }^{(10)}$

La encuesta evaluada buscó entregar a la comunidad científica un instrumento validado en Cartagena, Colombia, que permita evidenciar con certeza los conocimientos, actitudes y prácticas sobre el cuidado de menores de 5 años por parte de los padres o cuidadores, dada la importancia de estudiar estos conocimientos en los padres de niños de 0 a 5 años a fin de proyectar estrategias orientadas a la promoción de la salud en la primera infancia ${ }^{(11)}$.

La encuesta SIAC se tomó como un instrumento para obtener información objetiva sobre la evaluación de las actitudes, prácticas, conocimientos y dificultades respecto al cuidado de menores de 5 años, por parte de madres adolescentes. La información proporcionada es útil para el desarrollo de programas educativos y de apoyo referentes a la aplicación de la estrategia de AIEPI comunitario. Esta encuesta se 
puede utilizar para evaluar las necesidades individuales de las madres y los menores a su cargo, a fin de proporcionar apoyo individualizado según sea necesario (11)

Esta investigación logra obtener un instrumento que responde a las características de la población y al sistema de salud local, pudiendo ser utilizado en el contexto colombiano y Latinoamericano, pues se comparten rasgos culturales similares para la crianza de menores de 5 años ${ }^{(12)}$

A través del Análisis Factorial Exploratorio realizado, se determinaron los constructos del instrumento, para realizar este análisis se debe cumplir la aplicación de la prueba de adecuación muestral (KMO), la cual indica el grado de intercorrelación de las variables, si es mayor que 0,7 se considera factible y para la prueba de esfericidad de Barlett, se considera adecuada un valor de $p<0.05$ (6).

Se encontró que, dentro de estos 7 dominios resultantes, 2 arrojaron un KMO inferior a 0,60 , lo cual indicó que no eran válidos para el análisis factorial por componentes principales, por lo que se procedió a utilizar el método de extracción por ejes principales, ya que este es un método recursivo semejante a componentes principales, que permite justificar el análisis debido a que busca una mayor interrelación en cada factor ${ }^{(13)}$.

Este método permitió mirar la interrelación que poseen las variables entre sí, reduciendo las variables a un número menor, resultando suficiente para explicar los dominios o prácticas evaluadas, eliminando así todas las preguntas que no permitan medir de forma clara cada uno de los dominios ${ }^{(14)}$.

El análisis se desarrolló de forma individual para cada dominio, esto debido a que la estructuración de la encuesta no sigue un constructo especifico, sino que se apoya en varios conocimientos que nutren el trabajo comunitario a través de 18 prácticas claves que forman parte de la estrategia AIEPI, por lo cual se hizo necesario validar cada constructo de manera individual ${ }^{(5)}$.

Las prácticas de saneamiento básico, lactancia materna, inmunización, signos de alarma, cuidado de la mujer gestante, VIH/SIDA y prevención de accidentes, como componentes básicos de la estrategia AIEPI que fueron sometidas al análisis factorial exploratorio, permiten obtener un conocimiento del entorno sociocultural que puede influir en los estilos de crianza de los hijos, debido en parte a las creencias sobre ciertos aspectos de la vida diaria (entorno físico, alimenticios y de salud), que pueden ser un factor de riesgo en la morbilidad y mortalidad que involucra a los niños y niñas menores de 5 años ${ }^{(15)}$

El instrumento validado logró reducir la encuesta SIAC a 24 ítems para medir las practicas contenidas en dicha encuesta, permitiendo evaluar la actitud y conocimientos de las madres adolescentes sobre el cuidado de los menores de 5 años, de una forma sencilla y con un bajo costo ${ }^{(14)}$

No fue posible realizar un análisis factorial confirmatorio para investigar a priori la estructura multifactorial de cada escala, pues el estudio solo realizo una aplicación del instrumento.

Esta encuesta, se convierte en el primer instrumento validado en la Región Caribe y en Colombia para evaluar el componente comunitario de la Estrategia AIEPI, aspecto 
que se constituye en la principal fortaleza del estudio, permite además que sea aplicado en el contexto y evalúa el componente con menos ítems que la encuesta original, haciéndolo más eficiente en términos de tiempo y recursos económicos.

Se sugiere que en futuras investigaciones en la que se pretenda realizar la validación de este instrumento, se vincule la participación de madres de diferentes grupos etarios, para así tener una visión más amplia del mismo. Este aspecto podría constituir una limitación en la aplicación de sus resultados en contextos diferentes.

\section{CONCLUSIONES}

Los resultados obtenidos ponen de manifiesto que la encuesta SIAC que resultó del proceso de validación, mantuvo en los 7 dominios buenos niveles de validez después del análisis; sin embargo, es necesario evaluar los puntos de corte que permitan un diagnóstico más sensible y específico del grado de apropiación y aplicación de las practicas claves de la estrategia AIEPI, por parte de los padres o cuidadores de los niños y niñas menores de 5 años.

De igual manera, se logró evidenciar que el instrumento es una herramienta útil y necesaria para la evaluar cambios en los conocimientos, actitudes y prácticas de crianza de los padres y cuidadores, especialmente en aspectos como las medidas de higiene y saneamiento básico, lactancia materna y alimentación complementaria, inmunizaciones, identificación de signos de alarma, prevención de accidentes, cuidado de la mujer gestante y VIH/SIDA, aspectos importantes en torno a la salud de la primera infancia.

\section{REFERENCIAS}

1. Organizacion Mundial de la Salud. Salud de la madre, el recien nacido, del niño y del adoslecente. Suiza: Organizacion Mundial de la Salud; 2017. Disponible en: https://www.who.int/maternal child adolescent/topics/child/es/

2. Tatamues Chenas W. Enfermedades prevalentes en niños del centro infantil caritas alegres de la parroquia Tufiño-Tulcán, 2016 [Trabajo de Grado previo a la obtención del título de Licenciado en Enfermería] Ibarra: Universidad Tecnica del Norte. Facultad ciencias de la salud; 2019.

3. Pinzon E, Moran L, Loboa N, Cedeño P. Manejo clinico de pacientes menores de 5 años hospitalizados con un diagnostico de neumonia acorde con la estrategia AEIPI, en una institucion de salud, Cauca, Colombia. Revista Colombiana Salud Libre [Intenet] 2015 Diciembre 2015; 10 (5): 1-1 [Consultado Abril 1 2020] Disponible en: https://revistas.unilibre.edu.co/index.php/rcslibre/article/view/1431

4. Celis Callejas A, Delgado Martinez Y. Comportamiento parasitario, en niños menores de 5 años, del hogar carrusel de mis sueños en el segundo periodo del año 2017 [Proyecto para optar el título de especialista en epidemiología] Pereira: Fundacion Universitaria del Area Andina. Facultad de ciencias de la salud; 2017.

5. Chico M, Drasbek C, Benguigui Y. Guía para realizar encuestas de hogares sobre prácticas clave que salvaguardan la salud infantil / Guía para realizar la Encuesta de Hogares Sobre las Prácticas Clave que Protegen la Salud Infantil. Manejo Integrado de Enfermedades Infantiles (IMCI): Componente Comunitario / Atención Integrada a las Enfermedades Prevalentes de la Infancia (AIEPI): Componente Comunitario. 04.4 ed. [Internet] 1: Washington: Pan American Health Organization / World Health Organization; 2004 [Enero 2004; Consultado 2020 Abril 1] Disponible en: 
https://www.researchgate.net/publication/303289115 Guide for Conducting Househ old Surveys for Key Practices that Safeguard Child Health Guia para Realizar I a Encuesta de Hogares Sobre las Practicas Clave que Protegen la Salud Infan til Integrated Manag

6. Ministerio de salud y proteccion social. Lineamiento para el manejo integrado de la desnutrición aguda moderada y severa en niños y niñas de 0 a 59 meses de edad. Colombia: Ministerio de salud y proteccion social; 2017. Disponible en: https://www.minsalud.gov.co/sites/rid/Lists/BibliotecaDigital/RIDE/VS/PP/lineamientodesnutricion-aguda-minsalud-unicef-final.pdf

7. Carvajal A, Centeno C, Watson R, Martinez M, Sanz A. ¿Como validar un instrumeto de medida de la salud?. Anales Sis San Navarra [Internet] Pamplona 2011; 34 (1): 11 [Consultado 2020 Abril 1] Disponible en: http://scielo.isciii.es/scielo.php?script=sci arttext\&pid=S1137-66272011000100007

8. Vergara L. Practicas de Crianza en la primera infancia en los municipios de Riosucio y Manzanares. Zona proxima [Internet] 2017; 27 (1): 1-1 [Consultado 2020 Abril 1] Disponible http://rcientificas.uninorte.edu.co/index.php/zona/article/viewArticle/8412

9. Segredo A, Perez J, Lopez P. Construccion y validadcion de un instrumento para evaluar un clima organizacional en el ambito de la salud publica. Rev Cubana Salud Publica [Internet] 2015; 41 (4): 1-1 [Consultado 2020 Abril 1] Disponible en: http://scielo.sld.cu/scielo.php?script=sci arttext\&pid=S0864-34662015000400004

10. Carrillo G, Sanchez B, Vargas E. Desarrollo y pruebas psicometricas del instrumento "cuidar" - version corta para medir la competencia de cuidado en el hogar. Revista Salud UIS [Internet] 2016; 48 (2): 1-1 [Consultado 2020 Abril 1] Disponible en: https://revistas.uis.edu.co/index.php/revistasaluduis/article/view/5509

11. Cupe A, Garcia C. Conocimientos de los padres sobre la salud bucal de niños preescolares: desarrollo y validación de un instrumento. Rev Estomatol Herediana [Internet] 2015; 25 (2): 112-121 [Consultado 2020 Abril 1] Disponible en: http://www.scielo.org.pe/pdf/reh/v25n2/a04v25n2.pdf

12. Ramada J, Serra C, Delclos G. Adaptación cultural y validación de cuestionarios de salud: revisión y recomendaciones metodológicas. Salud Publica Mex [Internet] 2013; 55 (1): 1-1 [Consultado 2020 Abril 1] Disponible en: http://www.scielo.org.mx/scielo.php?script=sci arttext\&pid=S003636342013000100009

13. Caycho T. Importancia del análisis de invarianza factorial en estudios comparativos en Ciencias de la Salud. Educ Med Super [Internet] 2017; 31 (2): 1-1 [Consultado 2020 Abril 1] Disponible en: http://scielo.sld.cu/scielo.php?script=sci arttext\&pid=S0864-21412017000200004

14. Higuita L, Jaramillo A, Cardona J. Construcción de una escala sobre acepciones, percepciones y sensaciones durante la ECOE en estudiantes de Medicina. Educación Medica Superior [Internet] 2019; 33 (3): 1-1 [Consultado 2020 Abril 1] Disponible en: http://www.ems.sld.cu/index.php/ems/article/view/1730

15. Ortega J.Representación social de un grupo de cuidadores en la ciudad de Bogotá frente rol del psicólogo en las pautas de crianza, 2020

16. Vieira C, Enders B, Coura A, Menezes D, Lira L, Madeiros C. Validación de instrumento para la detección de adolescentes con sobrepeso en la escuela. Enfemer. glob. [Internet] 2016; 15 (43): 1-1 [Concultado 2020 Abril 1] Disponible en: http://scielo.isciii.es/scielo.php?script=sci arttext\&pid=S1695-61412016000300013 
